\title{
Prototypeneffekte im Grenzbereich von Phonologie und Morphologie
}

\author{
Hilke Elsen (München)
}

\begin{abstract}
This article presents names in science fiction and fantasy stories with problematic morphological structures as well as manufactured names which produce sound symbolic effects. The examples show a continuum from sound shape to recurring groups of sounds to morphemes. Not only individual phonemes, but also gestalts seem to constitute a level in language processing. Psychologically oriented approaches, e.g. prototype theory, deal with exactly these problems, structured categories, good and bad examples as well as continua between categories, even sound symbolism. They try to find systematicity where traditional linguistic approaches fail.
\end{abstract}

\section{$1 \quad$ Problemstellung}

In Science Fiction- und Fantasy-Geschichten treten einerseits viele Namen auf, die nicht in die üblichen morphologischen Wortbildungskategorien einordbar sind und unterschiedliche Grade an morphologischer Struktur aufweisen. Andererseits werden viele Kunstwörter nicht willkürlich für bestimmte Referentengruppen eingesetzt. Auch die Lautstruktur kann offenbar Hinweise auf die Referenz eines unbekannten Wortes geben, wenn keine anderen Informationen zur Verfügung stehen. Inter- und intrakategorielle Struktur widerspricht der Vorstellung von klaren Grenzen zwischen Kategorien und dem gleichen Stellenwert aller Mitglieder einer Kategorie. Auf beide Probleme der traditionellen Kategorisierungsprinzipien gehen Ansätze psychologisch orientierter Modelle im Zusammenhang mit der Prototypentheorie ein. In diesem Beitrag soll nun am Beispiel eines klar definierten Lexikonausschnitts gezeigt werden, wie wichtig die Auseinandersetzung mit diesen Problemen und die Suche nach adäquaten Lösungsmöglichkeiten ist. Der Artikel baut auf Material auf, das in Elsen (2008) publiziert wurde, und entwickelt dieses weiter.

\section{Die Beispiele}

\subsection{Wortbildung}

Bei einer empirischen Studie ${ }^{1}$ zu den Namen in Science Fiction- und Fantasy-Romanen wurden aus 52 deutschsprachigen Büchern die Namen aussortiert und nach Referentengruppe und morphologischer Bauweise untersucht (vgl. Elsen 2007c, 2008). Eine sehr große Gruppe

\footnotetext{
1 Das Projekt Phantastische Namen wurde am Institut für Deutsche Philologie der Ludwig-Maximilians-Universität München durchgeführt und dankenswerterweise mit Drittmitteln unterstützt von der Fa. Bau + Plan, München, und der DFG. Einige Autoren der untersuchten Bücher sind Hans Joachim Alpers, Andreas Brandhorst, Clark Darlton, Andreas Eschbach, Monika Felten, Herbert Franke, Helga Glaesener, Wolfgang Hohlbein, Wolfgang Jeschke, Hanns Kneifel, Kurd Laßwitz, Thomas Mielke, Helmut Pesch, Paul Scheerbart, Christiane Zina. Auf eine vollständige Quellenliste muss aus Platzgründen leider verzichtet werden.
} 
bilden Wortgruppenlexeme (vgl. Elsen 2007a) (1) und Komposita (2). Es handelt sich meist um sprechende Namen.

Graue Stadt, Sprechende Steine, Alter Bezirk, Kochender Fluß, Brauner Fluß, Gerstens Stern, Kugelsternhaufen M-13, Ganjasisches Reich, Hotel Leberecht, Boszyks Stern, Golf von Prem

Perlenmeer, Jamachimfluß, Eldermünde, Überwelt, Geistersümpfe, Staubwüste, Goshunsee, Waldwelt, Urirr-System, Saturn-Hotel

Daneben treten verschiedene Arten der Kurzwortbildung auf, und zwar bei Namen für Gruppierungen, Organisationen bzw. Institutionen.

HUMU/Humanistische Union, ZGU/Zentralgalaktische Union, SafCF/Staatsamt für Computerfragen, Chrekt/Chrekt-Orn, Störtis/Störtebekers Erben, Grassammler/Meergrassammler.

Schließlich gibt es die Konversion wie bei der Währung Solar oder dem Namen eines Arztes Moribund und auch die seltenere Zusammenrückung (Ohnegnad, Name eines Anwalts), die Zusammenbildung (Individualverformer) und die Kontamination (Terranglo, Sprache).

Breit gefächert sind die verschiedenen Möglichkeiten der Derivation. Die Präfigierung, wie bei der Substanz Superstabil, und die Präfixoidbildung, wie bei Riesenalp, kommt selten vor. Einmal tritt ein Präfix direkt an ein Suffix, und zwar bei dem Namen der Währung Transtel. Ein andermal kommt es zu Kombinationen von Pseudopräfix, Kunstwort und Pseudosuffix: ein Autor benutzt wiederkehrende Laut- bzw. Buchstabengruppen, um Verwandtschafts- und Berufsbeziehungen bei Zwergen zu kennzeichnen. So tritt $-i$ bei den ersten Zwergen auf, wenn sie noch keinen besonderen Status aufweisen (Bregi, Fregi, Gregi), -orin versprachlicht den Status eines Fürsten (Gregorin, Belforin, Burorin, Fregorin) und hama- den eines Meisters (Hamabregorin, Hamafregorin, Hamagregorin).

\subsection{Kunstwortbildung}

Die Liste der reinen Kunstwörter ist nahezu endlos, deswegen soll nur ein kleiner Ausschnitt aufgeführt werden. Kunstwörter kommen z. B. als Namen für Städte und Dörfer vor (Sainu, Draglop, Arziak, Bassacutena, Nyrngor, Churkuuhl, Grangor, Belhanka), für größere Gebiete wie Sternenreiche oder Länder (Lacom, Akranzôr, Arianreth, Berseb, Elvinon, Heyatha, TunAmrad), Flüsse (Boyus, Rodasch, Vensor, Endar), Gestirne wie Monde, Sterne, Sonnen (Atrax, Babersaig, Yra, Cacham, Gharn, Oranda), aber auch Krankheiten (Gragra), Bauten (Madalla, Agrachuridion, Dorak Angrîmur), Raumschiffe (Ozymandias), Getränke (Yolai) und Speisen (Al-Keht). Ausgeprägt häufig tritt die Kunstwortbildung auf bei den Namen für fremde Spezies (Dramax, Chirian, Marut-Shakot, Shenri, Shyr, Taruf) oder Individuen (Gorx, An-Rukhbar, Sham Crton, Rengas, Pyrnamalei Lazu-Zoltral, Brobb, Ygan, Azanthul, Tihamér).

\section{Der Zusammenhang von Sprachsystem und Sprachgebrauch}

Die Anordnung weist bereits auf unterschiedliche Verhältnisse von etablierten Morphemen und Kunstwortanteilen hin. Dieser Übergang von Wortbildung zu Kunstwortbildung wurde bereits an anderer Stelle hervorgehoben (Elsen 2006, 2007c, 2008) und auch die Probleme, die sich für eine traditionelle Sprachbeschreibung dadurch ergeben. Nicht viele Sprachwissenschaftler befassen sich mit solchen Gelegenheitsbildungen, die nicht der Standardsprache angehören. Häufig wird zwischen zwei Ebenen getrennt, was sich in Begriffen wie Langue Parole oder Kompetenz - Performanz ausdrückt. Sie können jedoch auch als ineinander übergehend aufgefasst werden. Versuchen wir, uns das anhand eines Modells vorzustellen. Die erste Ebene besteht aus abstrahierten Kategorien, die durch gute und häufige Beispiele vertreten werden. Sie ist leicht vermittelbar, dient als Einstieg in die linguistische Theorie und arbeitet mit den üblichen Einheiten wie Nomen, Verb, Kompositum. Unser Modell nun 
ersetzt zum Beispiel Frequenz durch unterschiedliche Grade an Helligkeit. Die häufigen und guten Beispiele werden in einer bildlichen Vorstellung durch eine dunkle Darstellung repräsentiert. Ausnahmen und seltene Beispiele, die entsprechend blass aussehen, ließen sich durch einen Filter leicht ausblenden.

Jetzt stellen wir den Filter so ein, dass nur Beispiele und Kategorien, die zu, sagen wir, $95 \%$ vorkommen, übrig bleiben. Es ergeben sich klar voneinander abgegrenzte Bereiche. Diese guten Beispiele finden einen Widerhall in der Repräsentation im Gehirn in Form von dichten Neuronenverbänden. Die "blassen" Ausnahmen sind entsprechend gering aktiviert und bestehen aus nur leicht vernetzten Verbänden, die sich auch schnell wieder auflösen können, denn Kategorisierungen und Häufigkeitseffekte sind auch neurologisch verständlich, da sie die Informationsverarbeitung erleichtern (Müller, Weiss 2000; mehr zu Netzwerken in Elsen 1999). Das Schaubild ist schwarz-weiß. Dies wäre der Bereich, dem sich zahlreiche Sprachwissenschaftler widmen, wenn das Sprachsystem, die Langue, den Forschungsgegenstand bildet. Beispiele der Parole wie Sprechervarianten oder Okkasionalismen, aber auch viele der phantastischen Namen aus der Untersuchung, fallen nun weg. Unser Grammatikbild ist übersichtlich und sauber. Die Kategorien werden als diskret empfunden.

Bei Bedarf wählen wir aber die $100 \%$-Einstellung, dazu eine Vergrößerung, und sehen uns die grauen, nun abgestuften Bereiche an. Dies ist die zweite Ebene des Modells und der Untersuchungsbereich der vorliegenden Studie. Beide Ebenen gehören zusammen. Veränderungen in den hellgrauen Bereichen können durchaus zu Veränderungen in den dunkelgrauen Zonen führen, wenn eine seltene Erscheinung immer häufiger auftritt und/oder als markant gilt.

\section{Strukturelle Übergangserscheinungen}

Auf der zweiten Ebene gibt es keine Liste mit gleichberechtigten Definitionskriterien mehr, die zu separaten Kategorien führen und alle Beispiele klar und problemlos zuordbar machen. Stattdessen gibt es gute Beispiele einer Kategorie und Zonen des Übergangs mit mehr oder weniger guten Vertretern, die die Kriterien mehr oder weniger gut aufweisen. Der Übergangsbereich führt hin zu guten Beispielen anderer Kategorien. Dies wird im Folgenden mit Tabellen dargestellt.

\begin{tabular}{|lcl|}
\hline Beispiel & $\begin{array}{l}\text { morphologisch } \\
\text { strukturiert }\end{array}$ & $\begin{array}{l}\text { bestehend aus vorhandenen, } \\
\text { bedeutungstragenden } \\
\text { Einheiten }\end{array}$ \\
\hline Perlenmeer & ja & ja + ja \\
\hline Jamachimflu $\beta$ & ja & nein + ja \\
\hline An-Rukhbar & ja & nein + nein \\
\hline Gorx & nein & nein \\
\hline
\end{tabular}

Tabelle 1: Eigenschaften von Komposita und Kunstwörtern

Auf der ersten, der schwarz-weißen Ebene sehen wir in der Tabelle (1) nur die erste und letzte Zeile, die Parameter sind entweder ganz (nur ja-Antworten) oder gar nicht gegeben (nur neinAntworten). Perlenmeer ist ein Determinativkompositum, das aus Lexemen des Deutschen besteht, Gorx ist ein Kunstwort ohne morphologische Struktur. Die Zeilen dazwischen zeigen durch die systematische Mischung der Antworten den Übergangsbereich an: Jamachimfluß sieht aus wie ein Kompositum, allerdings ist das erste Glied kein Morphem. Bei An-Rukhbar wird die Zweigliedrigkeit zwar durch die Bindestrichschreibung angedeutet, aber beide Glie- 
der sind keine Morpheme. Auf dieser Ebene, die auch die Graubereiche zulässt, erkennen wir Struktur und damit Ordnung. Noch deutlicher wird dies in den Tabellen (2) und (3).

\begin{tabular}{|c|c|c|c|}
\hline Name & $\begin{array}{l}\text { morph. } \\
\text { struktur. }\end{array}$ & $\begin{array}{l}\text { besteht aus vorhandenen } \\
\text { bedeutungstrg. Einheiten }\end{array}$ & Kommentar \\
\hline Venusier & ja & $\mathrm{ja}+\mathrm{ja}$ & \\
\hline Transtel & ja & $\mathrm{ja}+\mathrm{ja}$ & Verstoß gegen Regeln \\
\hline $\begin{array}{l}\text { Thorwaler, Ertruser, } \\
\text { Taimoner }\end{array}$ & ja & nein $+\mathrm{ja}$ & Kunstwort existiert selbständig \\
\hline Hylailsch, xuturisch & ja & nein + ja & $\begin{array}{l}\text { Kunstwort existiert auch mit } \\
\text { anderen Suffixen, aber nicht } \\
\text { selbständig }\end{array}$ \\
\hline $\begin{array}{l}\text { Mauriden, } \\
\text { Ongennen, } \\
\text { Garianer, Noioniten }\end{array}$ & ja & nein + ja & $\begin{array}{l}\text { Kunstwort existiert nur in dieser } \\
\text { Form }\end{array}$ \\
\hline Merkuria, Terrania & ja & $\mathrm{ja}+$ nein & $\begin{array}{l}\text { wiederholte stabile Relation } \\
\text { zwischen Wurzel und ergänzter } \\
\text { Wurzel }\end{array}$ \\
\hline $\begin{array}{l}\text { Erosan, Energen, } \\
\text { Euphorit }\end{array}$ & ja & ja + nein & $\begin{array}{l}\text { nur hypothetische/assoziative } \\
\text { Relation zwischen Wurzel und } \\
\text { Pseudosuffix }\end{array}$ \\
\hline $\begin{array}{l}\text { Thorta, Garethi, } \\
\text { Skrota }\end{array}$ & ja? & nein + nein & $\begin{array}{l}\text { Kunstwort existiert selbständig + } \\
\text { Pseudosuffix }\end{array}$ \\
\hline $\begin{array}{l}\text { Terkonit, Molvedin, } \\
\text { Karisom }\end{array}$ & ja? & nein + nein & $\begin{array}{l}\text { Kunstwort existiert nur in dieser } \\
\text { Form + Pseudosuffix }\end{array}$ \\
\hline Hamabregorin & ja? & nein + nein + nein & $\begin{array}{l}\text { Pseudopräfix + Kunstwort + } \\
\text { Pseudosuffix }\end{array}$ \\
\hline $\begin{array}{l}\text { Gorx, Sainu, } \\
\text { Draglop }\end{array}$ & nein & nein & \\
\hline
\end{tabular}

Tabelle 2: Eigenschaften von Ableitungen und Kunstwörtern

Zwischen dem klaren Beispiel für Ableitung, Venus-ier, und den Kunstwörtern Gorx, Sainu und Draglop kommen verschiedene Mischungen dazu vor. So besteht Transtel zwar aus Morphemen, aber es sind zwei Affixe, die nicht miteinander kombiniert werden dürfen. Bei Thorwaler, Ertruser und Taimoner handelt es sich um er-Ableitungen von Kunstwörtern, die in den Texten selbständig auftreten. Bei xuturisch und Hylailsch gibt es die abgeleiteten Kunstwörter nicht eigenständig, aber in Kombination mit anderen deutschen Suffixen. Mauriden, Ongennen, Garianer und Noioniten treten nur in dieser Form auf. Bei Merkuria und Terrania handelt es sich um im Deutschen vorhandene Stämme, an die ein Element angehängt wird, das wie ein Suffix wirkt insofern, als es Namen von Hauptstädten bildet. Produktiv ist diese Einheit nur in Texten des Science Fiction und Fantasy. Bei Erosan etc. handelt es sich um bekannte Morpheme in Kombination mit Pseudosuffixen, bei Garethi und Thorta bzw. Terkonit etc. scheint eine Struktur von Stamm und Suffix vorzuliegen, jedoch sind beide Teile eines Wortes nicht als Morphem des Deutschen vorhanden. Hamabregorin, vgl. auch die Beispiele in 2.1, ist nur in den Texten eines Autors als morphologisch komplex interpretierbar, es besteht aus Kunstwortanteilen, die aufgrund ihrer Kombinatorik in verschiedenen bedeutungsverwandten Namen ähnliche Bedeutungsveränderungen hervorrufen. Das heißt, die morphologische Durchsichtigkeit und der Anteil an Morphemen verringert sich in der Tabelle schrittweise von oben nach unten. 
Dies gilt auch für die Tabelle (3).

\begin{tabular}{|c|c|c|c|}
\hline Name & $\begin{array}{l}\text { morph. } \\
\text { struktur. }\end{array}$ & $\begin{array}{l}\text { besteht aus vorhandenen } \\
\text { bedeutungstrg. Einheiten }\end{array}$ & Kommentar \\
\hline $\begin{array}{l}\text { Graue Stadt, } \\
\text { Kochender Flu } \beta\end{array}$ & ja & $\mathrm{ja}+\mathrm{ja}+\mathrm{ja}$ & \\
\hline Ganjasisches Reich & ja & nein $+j a+j a+j a$ & $\begin{array}{l}\text { Kunstwort, regulär abgeleitet, } \\
\text { flektiert + Lexem }\end{array}$ \\
\hline Golf von Prem & ja & $j a+j a+$ nein & \\
\hline Signum Kearneyis & ja & ja + nein & Fremdwort + Kunstwort \\
\hline Dorak Angrîmur & ja? & nein + nein & $\begin{array}{l}\text { keine semantische Stütze der } \\
\text { beiden Teile }\end{array}$ \\
\hline $\begin{array}{l}\text { Gorx, Sainu, } \\
\text { Draglop }\end{array}$ & nein & nein & \\
\hline
\end{tabular}

Tabelle 3: Eigenschaften von Ableitungen und Kunstwörtern

Graue Stadt und Kochender Flu $\beta$ als klar morphologisch strukturierte Wortgruppenlexeme stehen Kunstwörtern wie Gorx gegenüber. Bei Ganjasisches Reich, Golf von Prem und Signum Kearneysis tritt ein Kunstwortanteil auf, im letzteren Fall in Verbindung mit einem Fremdwort. Bei Dorak Angrîmur ist eine Aufgliederung von zwei Wörtern nur graphisch, beides sind Kunstwörter.

Eine andere Möglichkeit, das Kontinuum darzustellen und dabei die Linearität des Übergangs zu betonen, ist eine Skala in Gestalt eines Pfeils mit zwei Spitzen (Bild 1). Die Ebene 1 des Modells wird durch die Spitzen vertreten, die dazu gehörenden Beispiele sind eingerahmt.

\begin{tabular}{|c|c|c|c|}
\hline $\begin{array}{l}\text { Kun } \\
{[-\mathrm{m}} \\
{[-\mathrm{bc}}\end{array}$ & $\begin{array}{l}\text { wort } \\
\text { rpholog. Struktur] } \\
\text { eutungstr. Einheiten] }\end{array}$ & $\begin{array}{r}\text { morpholog. ko } \\
{[+ \text { morph }} \\
{[+ \text { bedeutungstrage }}\end{array}$ & $\begin{array}{l}\text { xes Wort } \\
\text { Struktur] } \\
\text { inheiten] }\end{array}$ \\
\hline Gorx & Terkonit & Thorwaler & Venusier \\
\hline$A n$ & ukhbar & Jamachimflu $\beta$ & Perlenmeer \\
\hline Dot & k Angrîmur & Ganjasisches Reich & Graue Stadt \\
\hline
\end{tabular}

Bild 1: Eigenschaften von Kunstwörtern und morphologisch komplexen Wörtern

Einzeldarstellungen der Übergänge der verschiedenen Wortbildungstypen zu den Kunstwörtern oder Übergänge zwischen Wortbildungstypen ergeben dann ein netzwerkartiges Zusammenspiel mit dunklen Bereichen innerhalb eines hell- bis dunkelgrauen Gebiets.

Bisher wurden Kategorien von Wortbildungstypen betrachtet, die Parameter waren rein morphologisch-struktureller Natur. Nun steht für eine der Kategorien, die der Kunstwörter, die Beobachtung im Vordergrund, dass bestimmte Lautkörper bestimmte semantische Aspekte unterschiedlich gut wiedergeben. Es geht also um Kategorien wie "Name einer wunderhübschen jungen Frau" und lautstrukturelle Parameter, also klangliche Eigenschaften, vertreten durch graphische Zeichen. 


\section{$5 \quad$ Grade der Repräsentativität}

Die morphologisch klaren Beispiele befinden sich an den Enden der Skala oder, anders ausgedrückt, im dunklen Bereich. Die Kunstwörter sind aus morphologischer Sicht eindeutig, da sie keine morphologische Struktur aufweisen. ${ }^{2}$

Nachdem es bei den verschiedenen Möglichkeiten der Kombination von Kunstwörtern und etabliertem Sprachmaterial zu Abstufungen kommt, stellt sich die Frage, ob auch die Kunstwörter geordnet werden können, ob demzufolge auf einer Etappe der Skala, hier einer der Endpunkte, bei genügend Vergrößerung Struktur erkennbar ist. Bei der Analyse des Materials wurden nämlich viele Wortschöpfungen gefunden, die nicht willkürlich gewählt schienen. Die Autoren schilderten mehr oder weniger intuitive Auswahlverfahren. Aufgrund des fehlenden sprachwissenschaftlichen Hintergrundes konnten sie den Erstellungsvorgang und die Entscheidung für einen bestimmten Namen nur schwer begründen. Auch vermischen sie Lautgut und Schrift. Oft probierten sie aus, bis ein Name "passte". Die Autorenkommentare (vgl. Elsen 2008) lieferten erste Anhaltspunkte - für böse Charaktere eignen sich dunkle Vokale und harte Konsonanten, für Krieger kurze, harte Namen ohne i. Gute Magier bekommen wohlklingende Namen durch milde, summende Konsonanten wie $m$ oder $n$ und mehrere Vokale. Für Frauennamen eignen sich viele $y, e e, e, o u$. $O$ klingt bedrohlich und finster, $n$ weich, $i$ kindlich, $y$ sieht exotisch aus. Seltene Buchstaben und Buchstabenkombinationen werden gern in den Namen fremdartiger Wesen verwendet. Aber selbst wenn sich die Autoren bei manchen Kriterien für gute oder schlechte Namen einig sind, stellt sich die Frage, wie das bei den Lesern/Hörern empfunden wird. Also musste die Meinung der Rezipienten überprüft werden. Die Antworten ergaben ein etwas klareres Bild.

In Anlehnung an Rosch (1973) wurden 106 muttersprachliche Probanden nach guten und schlechten Namen für Lebewesen befragt. Die Erhebungen fanden im SS 2007 und WS 2007/08 an der LMU München sowie in einem Ingenieurbüro ${ }^{3}$ statt. Das Alter der 93 StudentInnen lag im Wesentlichen zwischen 20 und 27, das der dreizehn Mitglieder des Ingenieurbüros zwischen 32 und 52. Die Testpersonen bekamen jeweils sechs Beispiele zur Auswahl als mehr oder weniger gute Namen für bestimmte Lebewesen. Alle Beispiele stammen aus den Quelltexten. Die von den Autoren für die jeweilige Gruppe tatsächlich gewählten Namen sind in den Tabellen 4 bis 8 mit einem Sternchen markiert. Auf die Fragen war mit einer Note zwischen 1 (passt sehr gut) und 7 (passt überhaupt nicht) zu antworten, wobei eine Note auch mehrfach gegeben werden durfte. Für die Auswertung wurden bei den Antworten jeweils die Durchschnittsnoten ermittelt. Die Aufgaben und Notenmittelwerte lauteten wie folgt (Tabellen aus Elsen 2008):

\footnotetext{
2 Ihre Zahl, gemessen in types, ist in der Studie äußerst hoch, im Standardlexikon allerdings nicht. Die schwarzen Bereiche der ersten Ebene müssen also nicht unbedingt aufgrund von Frequenzeffekten entstehen.

3 Der einzige Unterschied bestand darin, dass sich diese Gruppe durch Vorbehalte gegen die Note 1 auszeichnete, so dass die guten Noten dadurch im Gesamtschnitt etwas schlechter wurden.
} 
1. Wie gut passt der Name für eine wunderschöne junge Frau?

\begin{tabular}{ll}
\hline Beispiel & Note \\
\hline Alani $^{*}$ & 1,90 \\
Sartassa $^{*}$ & 2,54 \\
Valeron Veit & 4,56 \\
Olda & 4,58 \\
Klipp & 5,76 \\
Gorx & 6,64 \\
\hline
\end{tabular}

Tabelle 4: junge Frau

2. Wie gut passt der Name für ein grausames, hässliches, bösartiges Monster?

\begin{tabular}{ll}
\hline Beispiel & Note \\
\hline Chrekt-Orn $^{*}$ & 2,07 \\
Ghuzdan $^{*}$ & 2,32 \\
Eugalp* $^{*}$ & 2,87 \\
Ch'tuon $^{*}$ & 2,93 \\
Tik & 5,27 \\
Gina & 6,59 \\
\hline
\end{tabular}

Tabelle 5: bösartiges Monster

3. Wie gut passt der Name für einen kleinen Gnom mit hoher Stimme?

\begin{tabular}{ll}
\hline Beispiel & Note \\
\hline Krillri* & 1,52 \\
Cir $^{*}$ & 1,81 \\
Gurlo & 3,58 \\
Cromag & 4,09 \\
Nana & 4,34 \\
Raul Madsen & 5,74 \\
\hline
\end{tabular}

Tabelle 6: kleiner Gnom

4. Wie gut passt der Name für ein fremdartiges, liebenswertes Wesen mit drei Beinen?

\begin{tabular}{ll}
\hline Beispiel & Note \\
\hline N'ehetu* $^{\prime}$ & 2,21 \\
Mu'ati* $^{*}$ & 2,49 \\
Cir & 3,06 \\
Gnoorat & 3,35 \\
Cara & 3,78 \\
Mark Nord & 5,58 \\
\hline
\end{tabular}

Tabelle 7: fremdartiges Wesen 
5. Wie gut passt der Name für einen guten, sehr mächtigen Magier?

\begin{tabular}{ll}
\hline Beispiel & Note \\
\hline Galdalyn* & 1,56 \\
Salamir* & 1,85 \\
Cuul & 4,05 \\
Oderich Finck & 4,10 \\
Rrul'ghargop & 4,25 \\
Nana & 5,56 \\
\hline
\end{tabular}

Tabelle 8: mächtiger Magier

Anzumerken ist, dass die Fremdartigkeit der Figuren in (4) sich nicht allein auf Dreibeinigkeit, sondern allgemein auf Andersartigkeit gegenüber Humanoiden bezieht. So sehen die Jombuuraner N'ehetu und Mu'ati aus wie Fische. Die vier Namen der Monster beziehen sich auf unterschiedlich stark bösartige Wesen - der Dämon Eugalp hat eine lange, hagere Gestalt, dürre Finger, trägt ein verrottetes Gewand, hat einen giftigen Körper, weil er Leichengift in sich sammeln kann, und erscheint insgesamt nur mäßig gefährlich. Olda wurde zwar auch als Frauenname gebraucht, jedoch für eine ängstliche Nebenfigur ohne die Attribute wie "schön" oder "jung". Die erste Frage zielte jedoch nicht allgemein auf eine weibliche Person, sondern auf eine mit besonders ausgeprägten reizvollen Attributen ab.

Die Testpersonen urteilten über Namen mit vorgegebener Referenz in fiktiven Situationen und damit nicht über Konzepte oder Wörter, deren Bedeutungen mithilfe von Merkmalsbündeln beschreibbar sind. Sie bewerteten den gesamten Laut-/Schriftkörper, die Gestalt (vgl. Elsen 2007b, 2009). Die Antworten verteilten sich nicht willkürlich, sondern zeigten System. Die Probanden beurteilten Alani, Sartassa, Krillri, Cir, N'ehetu, Mu'ati, Galdalyn, Salamir und alle vier Monsternamen klar als gute Beispiele für die Charaktere, so, wie von den Autoren intendiert. Klipp, Gorx, Tik, Gina, Raul Madsen, Mark Nord und Nana passten eindeutig nicht. Die Testpersonen erkannten also die richtigen Namen, jedoch ohne die textuellen Zusatzinformationen erhalten zu haben. Sie entschieden aufgrund des Lautkörpers, repräsentiert durch die Schreibung. Weiterhin zeigen die Abstände in der Notengebung Grenzen an. Zwischen den beiden passenden Beispielen und den übrigen Namen in den Tabellen 4, 6, und 8 sind große Abstände in der Notengebung zu sehen. Auch in Aufgabe 2 gibt es einen starken Bruch, und zwar zwischen Monstern und Nicht-Monstern. In der Tabelle 7 bekommen die beiden besten Beispiele nicht so gute Noten, der Unterschied zu Cir, einem kleinen Wesen, ist weniger ausgeprägt.

Außer einigen etablierten Namen wie beispielsweise Gina, Nana, Mark, Nord handelt es sich um Kunstwörter. Die Probandenantworten belegen klar, dass sie sich nicht gleich gut für die beschriebenen Referenten eignen. Und da die Beispiele allesamt keine morphologische Struktur aufweisen, kann die Anordnung nur auf ihre lautlich-graphische Eigenschaften zurückzuführen sein.

Im nächsten Schritt wurden einige Probandengruppen nach ihren Gründen für die Entscheidungen gefragt, das heißt, sie mussten eine nachträgliche Analyse der Gestalt-Wörter vornehmen und die wesentlichen lautlichen bzw. graphischen Kennzeichen des guten Beispiels ermitteln. Es ging darum herauszufinden, welche Eigenschaften der Referenten durch welche lautlichen Merkmale der Namen reflektiert werden. Folgende Attribute kristallisierten sich als relevant heraus: der Finallaut, die Dominanz eines bestimmten Vokals, die Silbenzahl, Einoder Zweiteiligkeit des Namens, aber auch eine gehäufte Menge an Velaren oder primär geöffnete Silben. Ein $a$ am Ende eines Namens zeigt einen Frauennamen an. Velare erinnern an die Lautäußerungen gefährlicher Tiere wie Bären oder Löwen (vgl. auch Ohala 1994). Dabei klingt ein Einsilbler, wenn er geschlossen ist, besonders hart. Durch $i$-Dominanz, am 
Besten im Einsilbler, hört sich ein Name klein und harmlos an. Viele offene Silben und $a$ Dominanz lösen Vorstellungen von Macht und Potenz, aber im positiven Sinne, aus. ${ }^{4}$ Finale Vokale verweisen auf weibliche Figuren. Dies führt uns wieder zurück zu dem Prototypenansatz. Denn einfache Merkmalsbündel bedeuten zwar eine Beschreibungsmöglichkeit und einen ersten Schritt für das Verständnis, dazu der recht klare Fall der ersten Gruppe, sind aber nicht genau genug. Die Tabelle 9 stellt die Attributlisten zu den Namen für schöne junge Frauen zusammen (die Orthographie repräsentiert die Lautung).

\begin{tabular}{l|cccccccccc} 
Beispiel & \multicolumn{3}{|l}{ Finallaut } & \multicolumn{3}{c}{$\begin{array}{c}\text { dominante } \\
\text { Vokale }\end{array}$} & \multicolumn{2}{c}{ Silbenzahl } & viele Velare & $\begin{array}{c}\text { offene } \\
\text { Silben }\end{array}$ \\
& $\mathrm{V}$ & $\mathrm{C}$ & $a$ & $o, u$ & $i$ & 1 & 2 & $3^{5}$ & & \\
\hline Alani & + & - & + & - & - & - & - & + & - & + \\
Sartassa & + & - & + & - & - & - & - & + & - & + \\
Valeron Veit & - & + & & & & - & - & + & - & \\
Olda & + & - & & & & - & + & - & - & \\
Klipp & - & + & - & - & + & + & - & - & - & - \\
Gorx & - & + & - & + & - & + & - & - & + & -
\end{tabular}

Tabelle 9: Formale Merkmale der Namen für junge Frauen

Das Ergebnis bei den beiden besten und schlechtesten Beispielen ist bipolar bezogen auf den Finallaut, den Silbenschluss und die Silbenzahl. Die beiden mittelguten Namen liefern unklare Ergebnisse, repräsentiert durch fehlende +/- -Zuweisungen. Alani wurde als bestes Beispiel gewertet, was nicht heißen soll, dass Gorx kein Frauenname sein kann, sondern eben ein besonders schlechter. Der Hauptunterschied zwischen den besten und schlechtesten Namen liegt beim Finallaut und der Silbenzahl, $a$ als bevorzugte Laut verweist auf gut und damit automatisch weiblich (laut Auskunft der Probanden). Wie in den Tabellen 1 bis 4 werden die Übergänge anhand von mehr oder weniger geltenden Attributen veranschaulicht.

Bei den nun folgenden Beispielen tritt das Merkmal der Einteiligkeit hinzu, denn Namen, die wie die Zusammenstellung eines Vor- und Nachnamen aussehen, deuten auf einen Menschen, allerdings nur, wenn er keine ungewöhnlichen Laute/Buchstaben bzw. entsprechende Kombinationen aufweist. Ein unbekanntes und darum auffälliges Laut- bzw. Schriftbild, vor allem ein Apostroph in einem Namen, weist auf die Fremdartigkeit des Trägers hin.

\begin{tabular}{l|cccccccccccc} 
Beispiel & \multicolumn{3}{|l}{ Finallaut } & \multicolumn{3}{c}{$\begin{array}{c}\text { dominante } \\
\text { Vokale }\end{array}$} & \multicolumn{1}{c}{ Silbenzahl } & $\begin{array}{c}\text { viele } \\
\text { offene }\end{array}$ & Einteiligkeit & $\begin{array}{c}\text { auffällige } \\
\text { Lautung7 }\end{array}$ \\
& $\mathrm{V}$ & $\mathrm{C}$ & $a$ & $o, u$ & $i$ & 1 & 2 & 3 & & & & \\
\hline N'ehetu & + & - & - & & - & - & - & + & - & + & + & + \\
Mu'ati & + & - & & & & - & - & + & - & + & + & + \\
Cir & - & + & - & - & + & + & - & - & & & + & - \\
Gnoorat & - & + & & & - & - & + & - & + & & + & \\
Cara & + & - & + & - & - & - & + & - & & + & + & - \\
Mark Nord & - & + & & & - & - & + & - & & - & - & -
\end{tabular}

Tabelle 10: Formale Merkmale der Namen für fremdartige Wesen

${ }^{4}$ Eine griechisch-lateinische Phonotaktik, zu der auch eine geschlossene Finalsilbe gehört ohne <e>, so dass eine Schwa-Interpretation ausgeschlossen wird, impliziert im entsprechenden Zusammenhang überdies gut wirkende Medikamente (vgl. Elsen 2004, 2006).

5 Oder mehr.

6 Es wird vorausgesetzt, dass das postvokalische $r$ (vor allem nach $a$ ) vokalisch ausgesprochen wird und mit dem $a$ verschmilzt. Inwiefern der Gelenklaut $s$ die vorhergehende Silben schließt, ist umstritten.

${ }^{7}$ Bzw. Schreibung. 
Hier sind die vokalischen Aspekte weniger relevant (Tab. 10). Wesentlich für die Zuordnung ist die unbekannte Lautung bzw. Schreibung für die Fremdartigkeit, dargestellt durch das Hochkomma mitten im Namen, auch finales $u$ ist im Deutschen nicht weit verbreitet. Cara ist als Frauenname etabliert, er wird ausgeschlossen. Die Zweiteiligkeit in Kombination mit unauffälliger Lautung schließt Fremdartigkeit aus, da sie typischerweise für Menschennamen gilt. Fehlende Velare deuten auf Gutmütigkeit. Die beiden besten Beispiele bekommen bei dieser Aufgabe nicht ganz so gute Noten. Der Unterschied zu Cir, einem kleinen Wesen, ist weniger ausgeprägt, vielleicht, weil die Gutmütigkeit als Eigenschaft relevant ist und auch bei dem $i$ mitspielt und die Fremdartigkeit über auffällige Lautung/Schreibung nicht so stark ausgedrückt wird, wie es beispielsweise bei sehr komplexen Konsonantengruppen der Fall ist.

\begin{tabular}{|c|c|c|c|c|c|c|c|c|c|c|c|c|}
\hline \multirow[t]{2}{*}{ Beispiel } & \multicolumn{2}{|c|}{ Finallaut } & \multicolumn{3}{|c|}{$\begin{array}{l}\text { dominante } \\
\text { Vokale }\end{array}$} & \multicolumn{3}{|c|}{ Silbenzahl } & \multirow[t]{2}{*}{$\begin{array}{l}\text { viele } \\
\text { Velare }\end{array}$} & \multirow[t]{2}{*}{$\begin{array}{l}\text { offene } \\
\text { Silben }\end{array}$} & \multirow[t]{2}{*}{ Einteiligkeit } & \multirow[t]{2}{*}{$\begin{array}{l}\text { auffällige } \\
\text { Lautung }^{8}\end{array}$} \\
\hline & V & $\mathrm{C}$ & $a$ & $o, u$ & $i$ & 1 & 2 & 3 & & & & \\
\hline Galdalyn & - & + & + & - & - & - & - & + & - & + & + & - \\
\hline Salamir & - & + & + & - & - & - & - & + & - & + & + & - \\
\hline Cuul & - & + & - & + & - & + & - & - & & - & + & \\
\hline Oderich Finck & - & + & & & & - & - & + & & & - & + \\
\hline Rrul'gargop & - & + & & + & - & - & - & + & + & - & + & + \\
\hline Nana & + & - & + & - & - & - & + & - & - & + & + & - \\
\hline
\end{tabular}

Tabelle 11: Formale Merkmale der Namen für mächtige Magier

Ein wesentliches Erkennungsmerkmal ist hier (Tab. 11) offenbar die Silbenzahl und die $a$ Dominanz. Denn $a$ wurde von den Probanden wiederholt mit guten Charakteren in Verbindung gebracht. Velare deuten auf ein bösartiges Wesen hin, darum passt Rrul'gargop nicht, obwohl er durch seine Mehrsilbigkeit und vor allem die geschlossene Endsilbe ein möglicher Kandidat für einen durch griechisch-lateinische Phonotaktik implizierten einflussreichen und fähigen Magiernamen sein könnte. Es wurde jedoch nach guten Magiern gefragt. Tatsächlich ist Rrul'gargop übrigens ein sehr mächtiges und darum äußerst gefährliches Monster. Dem Namen Oderich Finck verhilft die nicht geläufige Buchstabenfolge <-nck> zu einem schlechten Mittelplatz. Nana ist ein bekannter Frauenname, so dass er ausgeschlossen werden kann.

\begin{tabular}{l|cccccccccccc} 
Beispiel & \multicolumn{3}{|c}{ Finallaut } & \multicolumn{3}{c}{$\begin{array}{c}\text { dominante } \\
\text { Vokale }\end{array}$} & \multicolumn{1}{c}{ Silbenzahl } & $\begin{array}{c}\text { viele } \\
\text { offene }\end{array}$ & Einteiligkeit & $\begin{array}{c}\text { auffällige } \\
\text { Lautung }\end{array}$ \\
& $\mathrm{V}$ & $\mathrm{C}$ & $a$ & $o, u$ & $i$ & 1 & 2 & 3 & & & & \\
\hline Krillri & + & - & - & - & + & - & + & - & + & & & + \\
Cir & - & + & - & - & + & + & - & - & & - & + & - \\
Gurlo & + & - & - & + & - & - & + & - & & + & + & - \\
Cromag & - & + & & & - & - & + & - & + & & + & - \\
Nana & + & - & + & - & - & - & + & - & - & + & + & - \\
Raul & - & + & & & - & - & - & + & - & - & - & - \\
Madsen & & & & & & & & & & & &
\end{tabular}

Tabelle 12: Formale Merkmale der Namen für kleine Gnome

Die Dominanz des $i$ ist bei den Namen für kleine, harmlose Wesen der ausschlaggebende Faktor (Tab. 12), wobei zwei $i$ in zwei Silben besser sind als eines in einer Silbe. ${ }^{9}$ Raul Madsen als zweiteiliger und Nana als Frauenname werden sowieso leicht ausgeschlossen.

\footnotetext{
8 Bzw. Schreibung.

9 Möglicherweise handelt es sich bei der Wirkung von $i$ um eine lautsymbolische Universalie (vgl. z. B. Ohala 1994, Jakobson/Waugh 1987: 187, Bartens 2000: 9, Masuda 2007).
} 


\begin{tabular}{|c|c|c|c|c|c|c|c|c|c|c|c|c|}
\hline \multirow[t]{2}{*}{ Beispiel } & \multicolumn{2}{|c|}{ Finallaut } & \multicolumn{3}{|c|}{$\begin{array}{l}\text { dominante } \\
\text { Vokale }\end{array}$} & \multicolumn{3}{|c|}{ Silbenzahl } & \multirow[t]{2}{*}{$\begin{array}{l}\text { viele } \\
\text { Velare }\end{array}$} & \multirow[t]{2}{*}{$\begin{array}{l}\text { offene } \\
\text { Silben }\end{array}$} & \multirow[t]{2}{*}{ Einteiligkeit } & \multirow[t]{2}{*}{$\begin{array}{c}\text { auffällige } \\
\text { Lautung }\end{array}$} \\
\hline & V & C & $a$ & $o, u$ & $i$ & 1 & 2 & 3 & & & & \\
\hline $\begin{array}{l}\text { Chrekt- } \\
\text { Orn }\end{array}$ & - & + & - & & - & - & + & - & + & - & + & + \\
\hline Ghuzdan & - & + & & & - & - & + & - & & - & + & \\
\hline Eugalp & - & + & & & - & - & + & - & & & + & - \\
\hline Ch'tuon & - & + & - & + & - & - & + & - & & & + & + \\
\hline Tik & - & + & - & - & + & + & - & - & & - & + & - \\
\hline Gina & + & - & & - & & - & + & - & & + & + & - \\
\hline
\end{tabular}

Tabelle 13: Formale Merkmale der Namen für bösartige Monster

Gina als Frauenname und Tik mit der Implikation "klein, harmlos" fallen für die Monster eindeutig fort (Tab. 13), so dass die vier anderen Namen übrig bleiben. Mit der besten Note schneidet dann der Monstername mit den eindeutigsten Attributen ab. Der finale Konsonant markiert [+MÄNNLICH] bzw. [-WEIBLICH], und Monster sind (üblicherweise) nicht weiblich. Viele Velare weisen auf Bösartigkeit hin, unbekannte Phonotaktik auf Unbekanntheit. Die Benotung der restlichen Namen ist nicht ganz eindeutig. Ein fehlendes $i$ weist auf einen Mangel an Harmlosigkeit und kleiner Köpergröße hin und damit indirekt wieder auf Gefährlichkeit.

Diese Kriterien sind mehr oder weniger als cues oder Hinweise zu sehen, anhand derer sich die Rezipienten orientieren, wenn sie die Namen zuordnen und Anhaltspunkte aus dem Kontext fehlen. Eine $i$-Dominanz reicht allein offenbar für die Zuordnung zu kleinen, harmlosen Wesen aus. Genauso klar spricht die Zweiteiligkeit in Kombination mit unauffälliger Lautung/Schreibung gegen einen Namen für fremdartige Lebewesen. Außerdem spielt die Zusammensetzung der Gruppe, innerhalb der zu wählen ist, eine Rolle. Namen wie Nana oder Gina fallen als geeignete Kandidaten für Monster oder kleine Gnome automatisch weg, weil sie als Frauennamen sowieso nicht passen, obwohl sie für ein Monster grundsätzlich nicht ausgeschlossen werden können. Aber wäre es dann nicht weiblich und sympathisch? Je nach Referentengruppe verlieren manche Kriterien an Relevanz - bei fremdartigen Wesen spielte offenbar der finale Laut keine Rolle. Insofern ergeben sich unterschiedliche Zusammenstellungen und Gewichtungen lautlich-orthographischer Kriterien. Häufig wird angenommen, dass der Prototyp die größtmögliche Anzahl typischer Merkmale aufweisen sollte (Bärenfänger 2002: 4). Hier deutet sich jedoch an, dass ein Merkmal auch allein wirkt, wenn es wichtig genug ist (Beispiel Krillri), was die Stützung durch weitere Kriterien natürlich nicht ausschließt. Damit gewinnt die Salienz neben der Frequenz als Faktor an Gewicht. Bei einem Modell, das unterschiedliche Grade der Repräsentativität darstellen soll, reicht die Menge typischer Merkmale nicht aus. Es muss auch das Gewicht einzelner Merkmale berücksichtigen und das Zusammenspiel der Beispiele innerhalb einer Gruppe und gegenüber anderen Gruppen. Zusätzlich werden Merkmale, die eindeutig nicht vorhanden sind, bewertet. Das heißt, das Ausschlussverfahren ist ebenfalls eine Strategie bei der Beurteilung. Und dies gilt nicht nur in der beschriebenen Testsituation, sondern auch beim Ersterwerb von Lexemen (vgl. Elsen 1995).

Innerhalb des Bereichs der Kunstwörter gibt es also mehr oder weniger gute Vertreter, bezogen auf eine bestimmte Referentengruppe. Das Modell mit den schwarz-grau-weißen Bereichen ist dreidimensional geworden. Ein Fleck auf der Fläche, der mehr oder weniger stark ausgeprägte morphologische Struktur repräsentiert, kann weiter differenziert werden nach lautlich-symbolischen Eigenschaften.

Die Auseinandersetzung mit Daten des Sprachgebrauchs führt oft zu Analyseproblemen. Manche Kategorien haben keine klaren Grenzen, es gibt unterschiedliche Grade an 
Kategorienzugehörigkeit, wie am Beispiel der Namen gezeigt wurde. Also kommt es zu Übergängen, die durch mehr oder weniger stark ausgeprägte strukturelle Definitionskriterien motiviert sind. Andere entstehen über unterschiedliche Grade der Angemessenheit. Gute und schlechte Beispiele ergeben sich über die Urteile der Sprachbenutzer. Das Ergebnis hängt von den Mitkonkurrenten und der Zusammensetzung der Gruppe ab. Es gibt Merkmale, die so ausgeprägt sind, dass sie allein bei der Entscheidung für ein gutes Beispiel genügen. Es haben also nicht alle Merkmale das gleiche Gewicht und das beste Beispiel muss nicht unbedingt dasjenige mit den meisten passenden Merkmalen sein. Das Ausschlussverfahren ist eine weitere Strategie: wenn klare passende Attribute nicht ausreichen, kann mithilfe fehlender wichtiger Eigenschaften entschieden werden.

Eine Ebene bei der Sprachverarbeitung bzw. bei der Beurteilung ist die Gestalt. Wenn keine sonstigen Angaben vorhanden sind, gewinnen die Sprachbenutzer beim "Verstehen" unbekannter Wörter nämlich aus der Lautstruktur Informationen.

\section{Schluss}

Sprecherbefragungen können Hinweise auf Verarbeitungsstrategien geben. Die Prototypeneffekte dienen als Hinweise auf mögliche verarbeitungsrelevante Eigenschaften und erfordern neue Beschreibungsmöglichkeiten, die herkömmliche Merkmalsdarstellungen um mehrdimensionale, dynamische Illustrationsmöglichkeiten erweitern sollen. Hier bieten die Prototypenansätze und auch neuere Richtungen wie die Kognitive Grammatik (u. a. Taylor 2002, Ungerer/Schmid 2006) neue Lösungsansätze, denn sie berücksichtigen das Zusammenspiel von Wortbildung, Sprecher und Sprechsituation sowie mögliche stilistische Wirkungen. Langue und Parole sind zwar zwei verschiedene Ebenen für die Sprachbetrachtung, aber sie interagieren, denn Veränderungen auf der einen können zu Veränderungen auf der anderen führen, wenn sich beispielsweise die Pseudomorpheme bei den Substanzen von der Werbesprache und der Chemiesprache auch bei den phantastischen Namen finden und sich vielleicht weiter ausbreiten. Phänomene auf Parole-Ebene können sich etablieren und uns zu einem Einblick in den statu nascendi neuer Strukturen und die Funktionsweise von Sprache in Gebrauch verhelfen. An einigen Beispielen aus dem Übergangsbereich von Wortbildung und Phonologie wurde gezeigt, dass es auch dort Systematizität zu finden gibt. Mehr Untersuchungen zu solchen Bereichen könnten uns vielleicht einmal dabei helfen, die Funktionsweise von Sprache und Sprechen besser zu verstehen.

\section{Literatur}

Ballmer, Thomas T. (1980): "Sprache in Science Fiction". In: Ermert, Karl (ed.): Neugier oder Flucht? Zu Poetik, Ideologie und Wirkung der Science Fiction. Stuttgart: 82-94.

Bärenfänger, Olaf (2002): "Merkmals- und Prototypensemantik: Einige grundsätzliche Überlegungen". Linguistik online 12/3: 3-17.

Bartens, Angela (2000): Ideophones and Sound Symbolism in Atlantic Creoles. Helsinki.

Brdar-Szabó, Rita/Brdar, Mario (2000): "Grammaticalization and the lexicon: Core-and-periphery model vs prototype approach". In: Mangasser-Wahl, Martina (ed.): Prototypentheorie in der Linguistik. Tübingen: 139-159.

Elsen, Hilke (1995): "Der Aufbau von Wortfeldern". Lexicology 1/2: 219-242.

Elsen, Hilke (1999): Ansätze zu einer funktionalistisch-kognitiven Grammatik. Konsequenzen aus Regularitäten des Erstspracherwerbs. Tübingen.

Elsen, Hilke (2004): Neologismen. Formen und Funktionen neuer Wörter in verschiedenen Varietäten des Deutschen. Tübingen.

Elsen, Hilke (2005): "Das Kunstwort". Muttersprache 2/2005: 142-149.

Elsen, Hilke (2006): "Pseudomorpheme - Fiktive Namen im Übergangsbereich von Phonologie und Morphologie". Muttersprache 3/2006: 242-248. 
Elsen, Hilke (2007a): "Wortgruppenlexeme - Beispiele aus Enzyklopädie, Zeitung, Baurecht und Wasserbau". Fachsprache. International Journal of LSP. 1-2/2007: 44-55.

Elsen, Hilke (2007b): "Gestaltverarbeitung". Deutsch als Fremdsprache 44: 162-165.

Elsen, Hilke (2007c): "Die Wortbildung der Eigennamen in fiktionalen Texten". Zeitschrift für Literaturwissenschaft und Linguistik 148: 184-197.

Elsen, Hilke (2008): Phantastische Namen. Die Namen in Science Fiction und Fantasy zwischen Arbitrarität und Wortbildung. Tübingen.

Elsen, Hilke (2009): "Die Rolle der Gestalt in der Sprachverarbeitung". In: Eibl, Karl/Mellmann, Katja (eds.): Gestalt (= Journal of Literary Theory 2/2): 209-229.

Elsen, Hilke (in Vorb.): "Between phonology and morphology". In Onysko, Alexander/ Michel, Sacha (eds.): Word Formation from Cognitive Perspectives. Berlin/New York.

Elsen, Hilke/Michel, Sascha (2007): "Wortbildung und Sprachgebrauch. Desiderate und Perspektiven einer etablierten Forschungsrichtung". Muttersprache 1/2007: 1-16.

Fónagy, Iván (1983): La vive voix. Essais de psycho-phonétique. Paris.

Friedrich, Hans-Edwin (1995): Science Fiction in der deutschsprachigen Literatur. Tübingen.

Jakobson, Roman/Waugh, Linda R. (1987): The Sound Shape of Language. Berlin etc.

Mangasser-Wahl, Martina (2000): "Roschs Prototypentheorie - Eine Entwicklung in drei Phasen". In: Mangasser-Wahl, Martina (ed.): Prototypentheorie in der Linguistik. Anwendungsbeispiele - Methodenreflexion - Perspektiven. Tübingen: 15-31.

Masuda, Keiko (2007): "The physical basis für phonological iconicity". In: Tabakowska, Elzbieta/Ljundberg, Christina/Fischer, Olga (eds.): Insistent Images. Amsterdam/Philadelphia: $57-71$.

Müller, Horst M./Weiss, Sabine (2000): "Prototypen und Kategorisierung aus neurobiologischer Sicht". In: Mangasser-Wahl, Martina (ed.): Prototypentheorie in der Linguistik. Anwendungsbeispiele - Methodenreflexion - Perspektiven. Tübingen: 55-71.

Ohala, John J. (1994): "The frequency code underlies the sound-symbolic use of voice pitch". In: Hinton, Leanne/Nichols, Johanna/Ohala, John J. (eds.): Sound Symbolism. Cambridge: 325-347.

Rosch, Eleanor H. (1973): "On the internal structure of perceptual and semantic categories". In: Moore, Timothy E. (ed.): Cognitive Development and the Acquisition of Language. New York/London: 111-144.

Rosch, Eleanor H. (1975): "Cognitive representations of semantic categories". Journal of Experimental Psychology: General. 104/3: 192-233.

Scherwinsky, Felix (1978): Die Neologismen in der modernen französischen Science Fiction. Meisenheim.

Siebold, Oliver (2000): Wort - Genre - Text. Wortneubildungen in der Science Fiction. Tübingen.

Stoll, Kai-Uwe (1999): Markennamen. Sprachliche Strukturen, Ähnlichkeit und Verwechselbarkeit. Frankfurt am Main.

Taylor, John R. (1995): Linguistic Categorization. Prototypes in Linguistic Theory. $2^{\text {nd }}$ ed. Oxford.

Taylor, John R. (2002): Cognitive Grammar. Oxford.

Ungerer, Friedrich/Schmid, Hans Jörg (2006): An Introduction to Cognitive Linguistics. $2^{\text {nd }}$ ed. London etc.

Voigt, Gerhard (1985): "Zur linguistischen Bestimmung des Markennamens". In: Kürschner, Wilfried/Vogt, Rüdiger (eds.): Grammatik, Semantik, Textlinguistik. Tübingen. 123-136. 